\title{
MHD mixed convection flow of a power law nanofluid over a vertical stretching sheet with radiation effect
}

\begin{abstract}
A similarity solution of the steady magnetohydrodynamic (MHD) mixed convection boundary layer flow due to a stretching vertical heated sheet in a power law nanofluid with thermal radiation effect is theoretically studied. The governing system of partial differential equations is first transformed into a system of ordinary differential equations. The transformed equations are solved numerically using the shooting method. The influence of pertinent parameters such as the nanoparticle volume fraction parameter, the magnetic parameter, the buoyancy or mixed convection parameter and the radiation parameter on the flow and heat transfer characteristics is discussed. Comparisons with published results are also presented.
\end{abstract}

Keyword: Magnetohydrodynamic; Mixed convection; Power law nanofluid; Radiation effect; Stretching vertical sheet 